\title{
Article
}

\section{Moiré Superconductivity and the Roeser-Huber Formula}

\author{
Michael Rudolf Koblischka ${ }^{(\mathbb{D})}$ and Anjela Koblischka-Veneva ${ }^{(\mathbb{D}}$ \\ Saarland University, Experimental Physics, P. O. Box 151150, D-66041 Saarbrücken, Germany. \\ * Correspondence: m.koblischka@gmail.com
}

\begin{abstract}
As shown previously, a relation between the superconducting transition temperature and some characteristic distance in the crystal lattice holds, which enables the calculation of the superconducting transition temperature, $T_{\mathcal{c}}$, based only on the knowledge of the electronic configuration and of some details of the crystallographic structure. This relation was found to apply for a large number of superconductors, including the high-temperature superconductors, the iron-based materials, alkali fullerides, metallic alloys, and element superconductors. When applying this scheme called Roeser-Huber formula to Moiré-type superconductivity, i.e., magicangle twisted bi-layer graphene (tBLG) and bi-layer $\mathrm{WSe}_{2}$, we find that the calculated transition temperatures for $\mathrm{tBLG}$ are always higher than the available experimental data, e.g., for the magic angle $1.1^{\circ}$, we find $T_{\mathcal{c}} \approx 4.2-6.7 \mathrm{~K}$. Now, the question arises why the calculation produces larger $T_{\mathcal{C}}$ 's. Two possible scenarios may answer this question: (1) The given problem for experimentalists is the fact that for electric measurements always substrates/caps are required to arrange the electric contacts. When now discussing superconductivity in atomically thin objects, also these layers may play a role forming the Moiré patterns. The consequence of such substrate-induced super-Moiré patterns is that the resulting Moiré pattern always will show a larger cell size, and thus, a lower $T_{\mathcal{C}}$ of the final structure will result. (2) A correction factor to the Roeser-Huber formalism may be required to account for the low charge carrier density of the tBLG. Here, we test both scenarios and find that the introduction of a correction factor $\eta$ enables a proper calculation of $T_{\mathcal{c}}$, reproducing the experimental data. We find that $\eta$ depends exponentially on the value of $T_{c}$.
\end{abstract}

Keywords: Moiré Superconductors; twisted bilayer graphene, $\mathrm{WSe}_{2}$, superconducting transition temperature, Roeser-Huber formula

\section{Introduction}

Moiré superconductivity, which was first demonstrated experimentally in 2018, involves creating large, periodic superstructures in 2D materials as compared to the atomic scale. The first sample belonging to this new family of superconductors was found when stacking two graphene layers together with a small misalignment angle, $\Theta \sim 1.1^{\circ}$, called also the first magic angle. This graphene stack is called twisted bilayer graphene or abbreviated tBLG [1,2]. The misalignment between the two graphene layers creates a Moiré pattern which has a spatial period, $a_{M}$, being a factor $1 / \Theta$ larger than the unit cell on the atomic level. Very remarkably, the observed superconducting transition temperatures, $T_{c}$, can be several degrees $\mathrm{K}$ high, and the transition temperatures are highly sensitive to the misaligment angle $\Theta$.

Since these first experimental reports, superconductivity in tBLG has been observed in ambient conditions [3-7] and under pressure [2] by other authors in the literature as well, including various angles around the magic angle, different charge carrier densities, and different thicknesses of the hexagonal boron nitride (abbreviated $\mathrm{h}-\mathrm{BN}$ ) layers on top and bottom of the tBLG [7]. The superconducting properties, including the critical fields and the superconducting parameters $\kappa, \lambda_{L}$ and $\xi$ of these samples, are well documented including a classification by Talantsev [8]. Furthermore, the superconductivity of a trilayer stack of graphene with a misalingment of $\pm 1.1^{\circ}$ was reported [9]. Arora et al. 
have combined the $\mathrm{tBLG}$ with a monolayer of $\mathrm{WSe}_{2}$ additional to the h-BN layers [10], and also a report of superconductivity in misaligned $\left(\Theta=1^{\circ}, 4^{\circ}\right)$ double layers of $\mathrm{WSe}_{2}$ [11] can be found in the literature, but the data provided concerning superconductivity are less convincing as mentioned also in a recent review [12]. As superconducting domes appear in the phase diagram at different charge carrier concentrations and are separated by insulators and ferromagnets, this topic is intensively investigated by band structure calculations [13-16].

It is important to note here that Moiré patterns can be formed also in cases when different types of 2D-layered materials are stacked together, with or without angular misalignment, or between a 2D layer and a substrate [17,18]. As result, the resulting Moiré lattice parameter, $a_{M}$, may be considerably larger than the original atomic unit cells of any ingredient. Several details of the mathematics of Moiré patterns were already presented in Refs. [19-22]. Thus, the stacking of various 2D-layered materials offers a versatile new way to control superconductivity in layered 2D-systems ("Moirésuperconductors"), the full potential of which has been barely explored yet [23]. Thus, to further investigate this field and unleash more possibilities to find new materials with higher $T_{\mathcal{C}}$ 's, a simple calculation procedure which can be included in machine-learning approaches, see, e.g., Refs. [24-28], is extremely useful.

As the lattice constant of the Moiré pattern is playing an important role for the observation of superconductivity, it is straightforward to follow this relation between superconductivity and the characteristic sample dimension in more detail. For hightemperature superconductors (HTSc), and later also for iron-based superconductors (IBS), fullerenes, elemental superconductors and metallic alloys, the Roeser-Huber fomula was developed to calculate the superconducting transition temperature, $T_{\mathcal{c}}$, which only requires to find a characteristic length of the sample crystallography, $x$, and some knowledge about the electronic configuration [29-36]. All this information may be found in existing databases. Using the Roeser-Huber formula, the $T_{c}$ of several superconducting materials could be calculated with only a small error margin, and recently, the approach was employed to predict $T_{\mathcal{C}}$ of metallic hydrogen with different crystal lattices [37]. In case of double-doped HTSc materials (e.g., the $\mathrm{Cu}-\mathrm{O}$-plane of $\mathrm{Bi}_{2} \mathrm{Sr}_{2} \mathrm{CaCu}_{2} \mathrm{O}_{8+\delta}(\mathrm{Bi}-2212$ ) doped by oxygen and by additional metal ions like $\mathrm{Y}$ or La), two characteristic doping patterns result, and the final $T_{c}$ of the materials is calculated as a Moiré-pattern of the two patterns [31]. Thus, it is only straightforward to apply this calculation scheme to the real Moiré superconductors, where a clear crystallographic relation is defined by the orientation of the tBLG and by the unit cell of the tBLG itself.

In the present contribution, we present the application of the Roeser-Huber equation to Moiré superconductivity using the existing literature data for comparison.

\section{Materials and Methods}

\subsection{Roeser-Huber model}

The basic idea behind this approach is the view of the resisitive transition to the superconducting state as a resonance effect between the superconducting charge carrier wave, $\lambda_{\mathrm{cc}}$, and a characteristic length, $x=\lambda_{\mathrm{cc}} / 2$, in the sample. The details of this were already discussed in Refs. [29,30,35]. The Roeser-Huber-equation, originally obtained for high- $T_{\mathcal{C}}$ superconductors, is written as

$$
\left[(2 x)^{2} 2 M_{\mathrm{L}}\right] n_{0}^{-2 / 3} \pi k_{B} T_{c}=h^{2}
$$

where $h$ is the Planck constant, $k_{B}$ the Boltzmann constant, $x$ the characteristic atomic distance, $T_{\mathcal{C}}$ the superconducting transition temperature, $M_{\mathrm{L}}$ the mass of the charge carriers, and $n_{0}$ is a correction factor describing the number of $\mathrm{Cu}-\mathrm{O}$-planes in the HTSc unit cell. For $\mathrm{YBa}_{2} \mathrm{Cu}_{3} \mathrm{O}_{7-\delta}$ with one $\mathrm{Cu}-\mathrm{O}$-plane per unit cell, we have $n_{0}=1$, and the compound $\mathrm{Bi}_{2} \mathrm{Sr}_{2} \mathrm{CaCu}_{2} \mathrm{O}_{8+\delta}(\mathrm{Bi}-2212)$ with $2 \mathrm{Cu}-\mathrm{O}$-planes per unit cell has $n_{0}=2$. Thus, $n$ for $\mathrm{tBLG}$ is taken to be $n=1$ as the two graphene layers at the magic angle give 
together one superconducting unit. A system corresponding to $n_{0}=2$ would be then a stack of two 2D layers like h-BN-tBLG-h-BN-tBLG-h-BN, where the two tBLG layers are separated by a h-BN layer. As charge carrier mass, we assume in a first approximation $M_{\mathrm{L}}=2 m_{e}$, corresponding to a Cooper pair.

An energy, $\Delta_{(0)}$, can be introduced via

$$
\Delta_{(0)}=\pi k_{B} T_{\mathcal{C}},
$$

which may correspond to the pairing energy of the superconductor. So we can write

$$
(2 x)^{2} \cdot 2 M_{\mathrm{L}} n_{0}^{-2 / 3} \cdot \Delta_{(0)}=h^{2} .
$$

Using eq. (2) and regrouping of the terms leads finally to

$$
\Delta_{(0)}=\frac{h^{2}}{2} \cdot \frac{1}{M_{\mathrm{L}}} n_{0}^{2 / 3} \cdot \frac{1}{(2 x)^{2}}=\pi k_{B} T_{c} .
$$

Now, the formalism described above requires only minor adapations to the case of tBLG and its derivatives: $n_{0}=1$ was already mentioned. The Moiré lattice constant, $a_{M}$, plays the key role to describe a Moiré superconductor, so the characteristic length corresponds to $x=a_{M}$.

For a proper comparison of the calculated data to the experiments, $T_{\mathcal{C}}$ in the RoeserHuber formalism is to be taken from resistance measurements as the maximum of the derivative, $\mathrm{d} R / \mathrm{d} T$, corresponding to the mean field transition temperature $T_{c}^{\mathrm{MF}}$, which also plays an important role for the fluctuation conductivity analysis as described in Refs. [38-40]. In the literature, $T_{c}$ is often derived often from $50 \%$ of the normal-state resistance, which is not necessarily the same as $T_{c}^{\mathrm{MF}}$, especially not in the case of a two-step transition. Both these definitions of $T_{\mathcal{c}}$ are distinct from the $T_{\mathcal{C}}$ used in the Uemura plot $[1,8,41,42]$, where the completed transition when reaching $R=0 \Omega$ is considered. Other authors also have used $T_{(\mathrm{BKT})}$, the Berezinskii-Kosterlitz-Thouless (BKT) temperature, which is well suited for describing the superconducting transition in 2D systems like the ones investigated here. Most of the approaches mentioned here have, however, problems to give a proper value of $T_{c}$ when the superconducting transition is very broad, shows a secondary step, does not reach $R=0 \Omega$ or when the deviation from the normal-state resistivity is difficult to be defined.

Thus, in the present work all the published resistance data were digitalized and the derivative, $\mathrm{d} R / \mathrm{d} T$, was plotted graphically to obtain values for $T_{\mathcal{C}}$ according to the demands of the Roeser-Huber formalism.

\subsection{Materials}

Figure 1a presents a Moiré superlattice of two graphene layers (blue, red) tilted by an angle of $5^{\circ}$ for clarity. The resulting lattice parameter, $a_{\mathrm{M}}$, is indicated by a black line. In Ref. [9], also a tri-layer structure was presented with the top and bottom layers tilted by $\pm 5^{\circ}$ with respect to the center layer. This situation is depicted in Fig. 1b.

The lattice parameter of graphene is $a_{0}^{G}=0.246 \mathrm{~nm}$, and the one of $\mathrm{WSe}_{2}$ is $a_{0}^{\mathrm{WSe}_{2}}=$ $0.353 \mathrm{~nm}$ [43]. Then, the possible Moiré patterns of two identical layers at an angle $\Theta$ have a periodicity according to

$$
a_{M}=a_{0} / 2 \sin (\Theta / 2),
$$

with $a_{M}$ denoting the lattice constant of the Moiré superlattice (MSL). Figure 1c depicts the dependence of the Moiré lattice constant, $a_{\mathrm{M}}$, as a function of $\Theta$ for graphene as well as for $\mathrm{WSe}_{2}$. The first magic angle, $1.1^{\circ}$, is indicated by a dashed green line.

To measure the superconducting properties of tBLG by means of resistance measurements, a structure called device is fabricated using the tear-and-stack or cut-and-stack method encapsulating the tBLG between h-BN layers. This arrangement is then pat- 
(a)

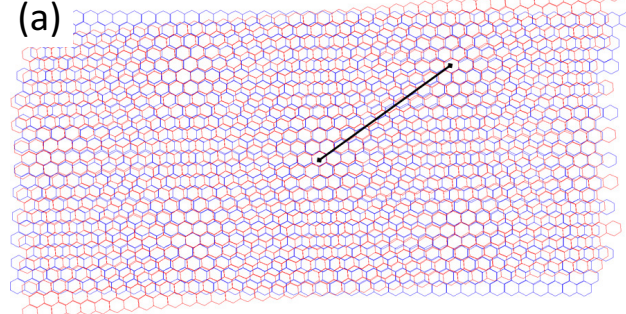

(c)

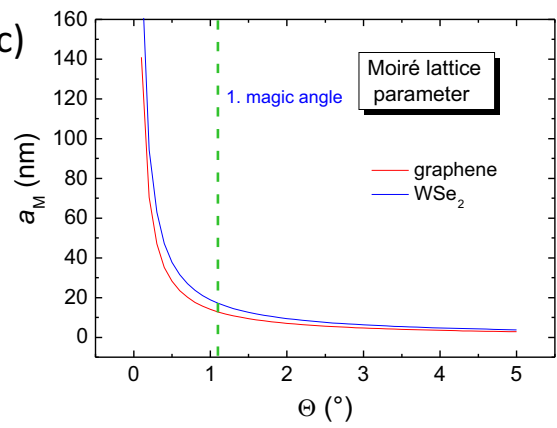

(b)

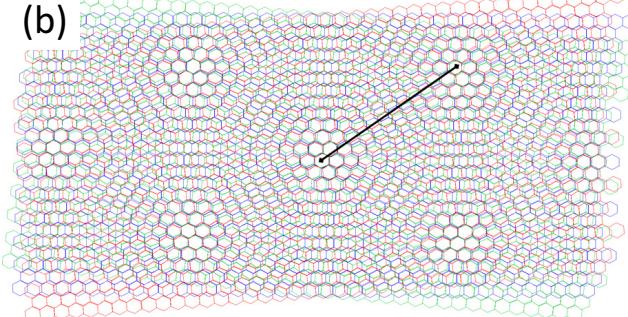

(d)

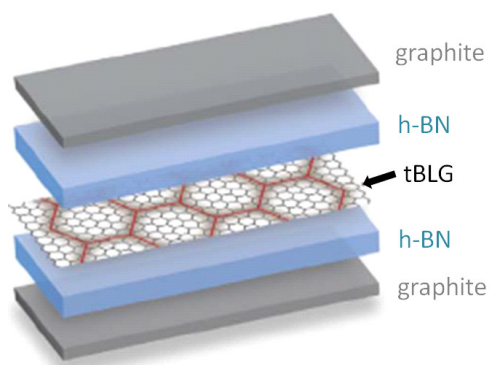

Figure 1. (a) Moiré pattern of two graphene layers (red, blue) tilted by $5^{\circ}$. This value was chosen for clarity. The black line indicates the resulting Moiré lattice parameter, $a_{\mathrm{M}}$. (b) Moiré pattern of a tri-layer graphene system (red, blue, green) with the top and bottom layer tilted by $\pm 5^{\circ}$ with respect to the center layer. (c) Moiré lattice parameter, $a_{\mathrm{M}}$, of graphene as function of the tilt angle, $\Theta$. The first magic angle, $1.1^{\circ}$, is marked by a dashed green line $(=-=-=)$. (d) Schematic view of the various layers in a device for resistance measurement. Figure adapted from Ref. [2].

terned into a Hall bar geometry with multiple leads using electron beam lithography and reactive ion-etching. The final device is placed on $\mathrm{Si} / \mathrm{SiO}_{2}$ substrate with an intermediate thick graphite layer serving as back gate. Another graphite layer on top serves for protection. This construction is required to prepare proper electric contacts to the sample. A schematic drawing of the arrangement of the various layers is given in Fig. $1 d$.

The accuracy achieved to determine the tilt angle of the graphene layers is typically $\sim 0.03^{\circ}$ [7]; Stepanov et al. describe the twist homogeneity within a device as good as $0.01^{\circ}$ per $10 \mu \mathrm{m}[6]$.

\section{Results and Discussion}

New results with much higher values of $T_{\mathcal{C}}$ were presented recently by Saito et al. [7], who also used the h-BN as top and bottom cover, but varied the tilt angle between $1.02^{\circ}$ and $1.20^{\circ}$ and the thickness of the h-BN layer between $6.7 \mathrm{~nm}$ and $68 \mathrm{~nm}$. These experiments demonstrated that the device (device 5) with a tilt angle of $\Theta=1.10-1.15^{\circ}$ and a h-BN thickness of $45 \mathrm{~nm}$ showed the highest $T_{c}$ ever reported for the tBLG systems. Stepanov et al. [6] also fabricated devices with varying the h-BN thickness between 7 and $12.5 \mathrm{~nm}$. Codecido et al. [4] showed superconductivity in tBLG at a much smaller angle $\Theta=0.93^{\circ}$, so superconductivity does exist in a wide range around the magic angle. Lu et al. [3] have shown a complete phase diagram of their tBLG sample with four domes of superconductivity at positive and negative charge carrier densities by plotting the measured longitudinal resistance versus temperature and charge carrier density, demonstrating the experimental advances since the first reports of superconductivity in tBLG.

These results provided the base to compare the Roeser-Huber calculations with a wider experimental dataset. For comparison, we employed the data of Saito et al. (their Fig. 3c), and those of Refs. $[1-6,10]$. The $T_{c, \text { opt }}$ determined by Saito et al. corresponds directly to $T_{c}^{\mathrm{MF}}$ required by us, so the data can be directly compared to each other as done in Table 1 below. Table 1 presents the $T_{c}$-values of several tBLG devices of various 


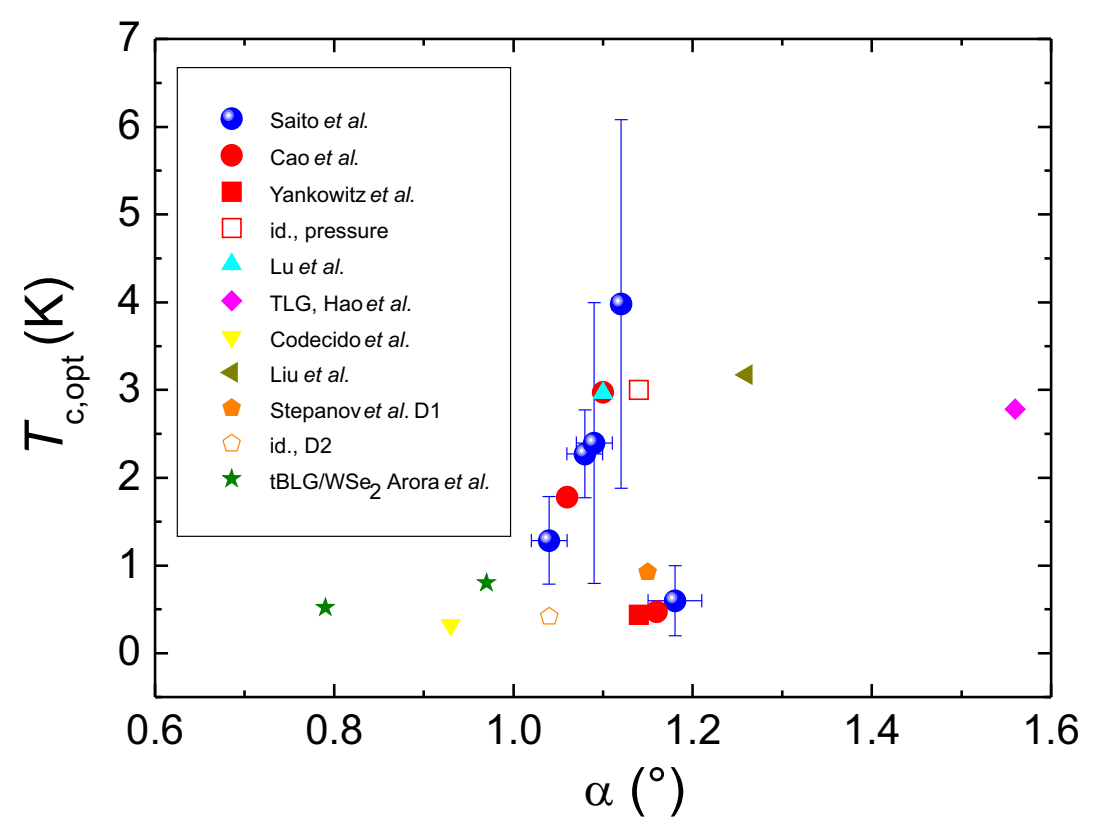

Figure 2. Experimental data for the superconducting transition temperature, $T_{\mathcal{c}}$, with the respective error bars. Data taken from Saito et al. (Ref. [7]), together with data of Cao et al. [1], Yankowitz et al. [2], Lu et al. [3], Liu et al. [5], Codecido et al. [4], Stepanov et al. [6] and Arora et al. [10].

authors [1-7] together with data of a graphene tri-layer [9], the data of $\mathrm{WSe}_{2}$-stabilized tBLG [10] and the data obtained on twisted $\mathrm{WSe}_{2}$ bi-layers [11]. Listed are the tilt angle $\alpha$, the experimentally determined value of $T_{\mathcal{c}}(\exp )$ corresponding to our definition of $T_{c}^{\mathrm{MF}}$, the characteristic length, $x$, corresponding to the Moire lattice constant $a_{\mathrm{M}}$, the energy $\Delta_{(0)}$ calculated using $n_{0}=1, M_{L}=2 m_{e}$ and the calculated values of $T_{\mathcal{c}}$ (calc). When doing the calculations, the calculated values $T_{c}$ (calc) turned out to be much larger as the experimentally observed values for $T_{c}$. The first two rows give the data for tBLG at the magic angle, $\Theta=1.1^{\circ}$, yielding $4.23 \mathrm{~K}$ with $n_{0}=1$. Using $n_{0}=2$ would lead to a $T_{c}$ of $6.714 \mathrm{~K}$, which is even higher and unrealistic. Table 1 shows further that the experimental variation of the tilt angle between $0.93^{\circ}$ (the smallest tilt angle reported for superconductivity in tBLG) and $1.18^{\circ}$ leads to $T_{c}$-values of pure tBLG ranging between $3.024 \mathrm{~K}$ and $4.867 \mathrm{~K}$. Thus, all calculated data are clearly higher than the experimental ones. What could be the reason for this?

There are two possible scenarios to explain this outcome.

(1) The effective Moiré lattice parameter is much larger as determined by eq. (5).

This is possible when considering the fact that Moire superlattices can be formed by all layers involved forming the device, not only the graphene bilayer as intended. The fully encapsulated graphene has necessarily two interfaces with the h-BN layers on the top and bottom, where an extra tilt can occur. Looking at Fig. 1c and eq. (5), the effect is largest at very small angles.Thus, attempting to align the top and bottom h-BN layer to the graphene may generate much larger Moiré superlattice parameters. Such a situation was discussed by Wang et al. [18].

In case of a stack of h-BN with graphene, there is a misfit between the two lattices, so the resulting superlattice can be described as $[2,20]$

$$
a_{\mathrm{MSL}}=\frac{(1+\delta) a_{0}}{\sqrt{2(1+\delta)(1-\cos \Phi)+\delta^{2}}}
$$

where $\delta$ denotes the lattice mismatch between h-BN and graphene (1.8\%) and $\Phi$ is the twist angle of h-BN with respect to graphene. A result of this is that the largest possible Moiré lattice constant is $\sim 14 \mathrm{~nm}$, which occurs when the one graphene layer is fully 
Table 1. Table giving the experimental data of $T_{\mathcal{C}}$, the angles and the resulting characteristic length, $x$, the calculated energy $\Delta_{(0)}$ and $T_{\mathcal{c}}$ (calc) using the Roeser-Huber equation (eq. 1 with $n=1$ and $M_{L}=2 m_{e}$. The energy $\Delta_{(0)}^{*}$ and the transition temperature $T_{c}^{*}$ (calc) are calculated using the correction factor $\eta$. Furthermore, the sample names of the original publication and the references are given. The $T_{c}$ marked by + is the value claimed by the authors from a two-step transition. Our $T_{c}$ determined from their data is $T_{\mathcal{c}}=0.32 \mathrm{~K}$. $\ddagger$ This value gives the zero resistance. Stars $\left(^{*}\right)$ mark the $W \mathrm{We}_{2} T_{c}$-data from the experiments of An et al. [11], where the $T_{c}$ values given are determined by us. $\left({ }^{\otimes}\right)$ as given by the authors for $R=0 \Omega$. $\left(^{* *}\right)$ indicates $T_{\mathcal{C}}$ determined via a $50 \%$ normal-state resistance criterion.

\begin{tabular}{|c|c|c|c|c|c|c|c|c|c|c|}
\hline type & $\begin{array}{l}\text { tilt angle } \alpha \\
{\left[{ }^{\circ}\right]}\end{array}$ & $\begin{array}{l}T_{\mathcal{C}}(\exp ) \\
{[\mathrm{K}]}\end{array}$ & $\begin{array}{l}x \\
{[\mathrm{~nm}]}\end{array}$ & $\begin{array}{l}\Delta_{(0)} \\
{\left[10^{-22} \mathrm{~J}\right]}\end{array}$ & $\begin{array}{l}T_{C}(\text { calc }) \\
{[\mathrm{K}]}\end{array}$ & $\begin{array}{l}\Delta_{(0)}^{*} \\
{\left[10^{-22} \mathrm{~J}\right]}\end{array}$ & $\begin{array}{l}T_{c}^{*}(\text { calc }) \\
{[\mathrm{K}]}\end{array}$ & $\eta$ & $\begin{array}{l}\text { comment/ } \\
\text { sample name }\end{array}$ & Ref. \\
\hline \multirow[t]{2}{*}{ tBLG } & 1.1 & - & 12.81 & 1.835 & 4.23 & - & - & - & $n_{0}=1$ & \multirow[t]{2}{*}{ magic angle } \\
\hline & 1.1 & - & 12.81 & 2.912 & 6.714 & - & - & - & $n_{0}=2$ & \\
\hline \multirow[t]{14}{*}{ tBLG } & 1.16 & 0.47 & 12.15 & 2.040 & 4.704 & 0.204 & 0.470 & 20 & M1 & Cao et al. \\
\hline & 1.05 & 1.7 & 13.42 & 1.671 & 3.854 & 0.740 & 1.705 & 4.52 & M2 & Cao et al. \\
\hline & 1.14 & 0.6 & 12.36 & 1.971 & 4.542 & 0.197 & 0.454 & 20 & D1 & Yankowitz et al. \\
\hline & 1.27 & 3 & 11.10 & 2.446 & 5.638 & 1.304 & 3.007 & 3.75 & $\begin{array}{l}\mathrm{D} 2 \\
(1.33 \mathrm{GPa})\end{array}$ & Yankowitz et al. \\
\hline & 1.08 & 2.27 & 13.05 & 1.768 & 4.877 & 0.982 & 2.265 & 3.6 & device 1 & Saito et al. \\
\hline & 1.09 & 2.395 & 12.93 & 1.801 & 4.153 & 1.044 & 2.408 & 3.45 & device 2 & Saito et al. \\
\hline & 1.04 & 1.29 & 13.55 & 1.639 & 3.781 & 0.561 & 1.295 & 5.84 & device 3 & Saito et al. \\
\hline & 1.12 & 3.98 & 12.58 & 1.902 & 4.385 & 2.606 & 3.986 & 2.2 & device 5 & Saito et al. \\
\hline & 1.18 & 0.6 & 11.94 & 2.111 & 4.867 & 1.792 & 0.601 & 16.2 & device 4 & Saito et al. \\
\hline & 1.1 & 0.25 & 12.81 & 1.835 & 4.23 & 1.287 & 2.968 & 2.85 & - & Lu et al. \\
\hline & 0.93 & $<0.5+$ & 15.16 & 1.311 & 3.024 & 0.139 & 0.32 & 18.9 & smallest $\Theta$ & Codecido et al. \\
\hline & 1.26 & $<3.5 \ddagger$ & 11.19 & 2.407 & 5.550 & 1.376 & 3.171 & 3.5 & - & Liu et al. \\
\hline & 1.15 & 0.92 & 12.26 & 2.005 & 4.632 & 0.401 & 0.925 & 10 & D1 & Stepanov et al. \\
\hline & 1.04 & 0.4 & 13.55 & 1.640 & 3.781 & 0.786 & 0.398 & 19 & D2 & Stepanov et al. \\
\hline TLG & 1.56 & 2.7 & 9.035 & 3.69 & 8.507 & 1.19 & 2.784 & 6.2 & - & Hao et al. \\
\hline \multirow[t]{2}{*}{$\mathrm{tBLG}+\mathrm{WSe}_{2}$} & 0.97 & 0.8 & 14.53 & 1.43 & 3.289 & 0.348 & 0.802 & 8.2 & D1 & \multirow[t]{2}{*}{ Arora et al. } \\
\hline & 0.79 & 0.52 & 12.73 & 0.946 & 2.182 & 0.225 & 0.520 & 8.4 & D3 & \\
\hline bi-layer & 1 & $3.32^{*}$ & 18.89 & 0.844 & $1.95\left(n_{0}=1\right)$ & - & - & - & E7, $-14.4 \mathrm{~V}$ & \multirow[t]{7}{*}{ An et al. } \\
\hline \multirow[t]{6}{*}{$\mathrm{WSe}_{2}$} & 1 & $3^{\otimes}$ & 18.89 & 1.340 & $3.09\left(n_{0}=2\right)$ & - & - & - &,-- & \\
\hline & 1 & $3^{\otimes}$ & 20 & 0.753 & $1.74\left(n_{0}=1\right)$ & - & - & - &,-- & \\
\hline & 1 & $3^{\otimes}$ & 20 & 1.195 & $2.76\left(n_{0}=2\right)$ & - & - & - &,-- & \\
\hline & 2 & $4.53^{*}$ & 9.45 & 3.376 & $7.78\left(n_{0}=1\right)$ & 1.963 & 4.53 & 3.44 & $F 2,-6.65 \mathrm{~V}$ & \\
\hline & 2 & $6.1^{*}$ & 9.45 & 3.376 & $7.78\left(n_{0}=1\right)$ & 2.648 & 6.11 & 2.55 & $\mathrm{~F} 2,-6.92 \mathrm{~V}$ & \\
\hline & 4 & $6(50 \%)^{* *}$ & 4.72 & 13.5 & $31.1\left(n_{0}=1\right)$ & - & - & - & D11, $-17.9 \mathrm{~V}$ & \\
\hline
\end{tabular}

aligned to the h-BN layer. Wang et al. showed that they can increase the MSL lattice parameter to $29.6 \mathrm{~nm}$ by aligning both $\mathrm{h}$-BN layers to the graphene. Calculating $T_{\mathcal{C}}$ with this MSL parameter would yield a value of $\sim 0.8 \mathrm{~K}$, which would be much closer to the experimental data.

However, the high pressure experiment of Yankowitz et al. [2] and the data of Saito et al. [7] demonstrated that this explanation cannot be the solution of the present problem. The optical images of the devices presented by Cao et al. [1], Yankowitz et al. [2] and Saito et al. [7] showed all arrangements made before putting the top h-BN layer in place. Thus, the misfit would be created when placing this layer. While this scenario might have applied to the first reports of superconducting tBLG, all authors of the more recent contributions have explicitly checked for such effects and even provided a dedicated discussion in their Supplementary Data (see, e.g., Fig. S2 of Ref. [3]), so this effect can be ruled out. Furthermore, the high-pressure experiment could increase $T_{\mathcal{C}}$ from $0.6 \mathrm{~K}$ to $3 \mathrm{~K}$ with the same configuration, and the data of Saito et al. [7] showed that their experimental values of $T_{\mathcal{c}}$ are approaching the calculated ones.

(2) A new correction factor must be introduced to the Roeser-Huber equation.

The band structure calculations have shown that the charge carrier density of the tBLG at 


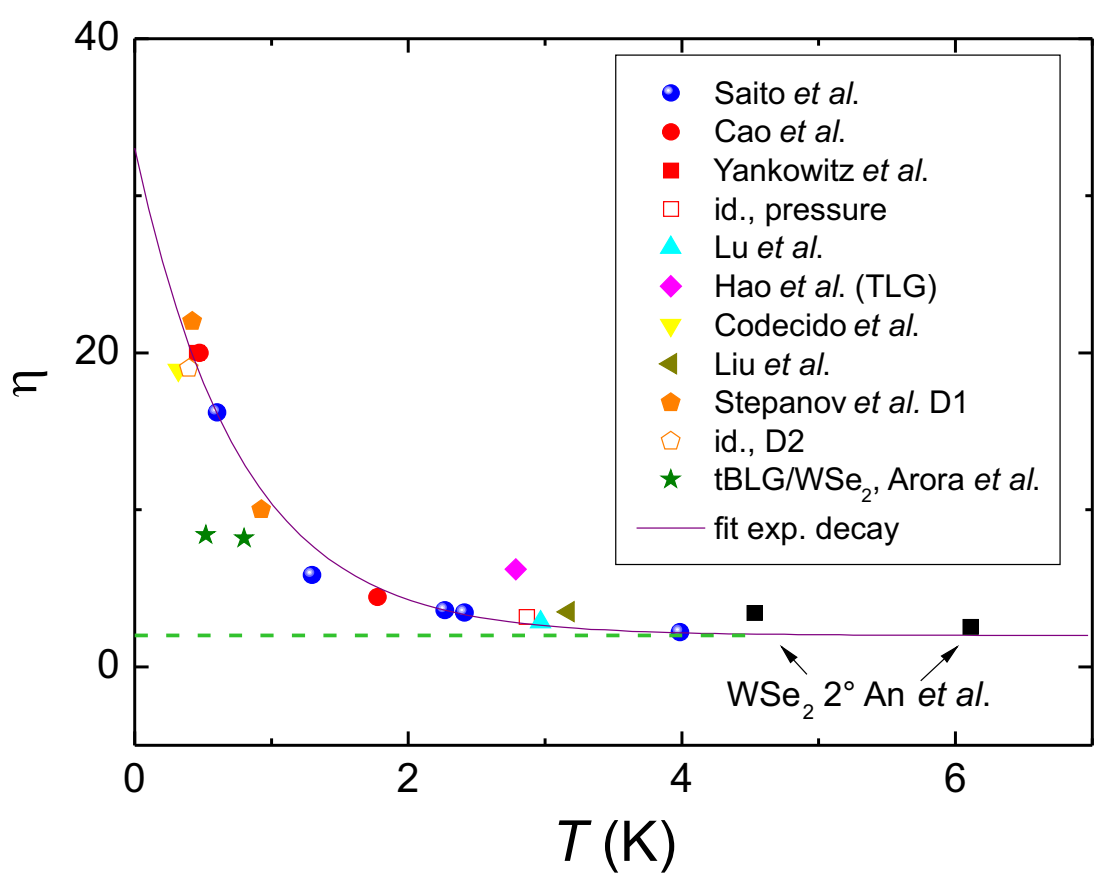

Figure 3. The correction factor $\eta$ as function of temperature. Included here are the tBLG data of Refs. [1-7], the trilayer graphene (TLG) of Hao et al. ( $\downarrow,[9])$, the tBLG/WSe 2 of Arora et al. ( $\star$, [10]) and the $2^{\circ} \mathrm{WSe}_{2}$ data of An et al. (ם, [11]). The violet line (-) is a fit to all data using eq. (8).

the magic angle is very small, and it is shown by Lu et al. [3] that several superconducting domes can be found when plotting the linear resistance versus carrier density and temperature, which equals a phase diagram of tBLG. Thus, this fact must be accounted for in our calculations. Cao et al. [1] showed that the effective mass of the charge carriers is only $0.2 m_{e}$, and in the Uemura plot (their Fig. 6), they demonstrated that the tBLG samples are located at low Fermi temperatures $T_{F} \approx 20 \mathrm{~K}$ and $n_{2 \mathrm{D}}=1.5 \times 10^{11} \mathrm{~cm}^{-2}$, being clearly distinct from the HTSc. writing:

Thus, we introduce a correction factor, $\eta$, to the charge carrier mass $M_{L}$ in eq. (1) by

$$
M_{L}=\eta m_{e} .
$$

The situation $\eta=2$ will then correspond to our initial value of 2. Now, we come back to Table 1 . The energy $\Delta_{(0)}^{*}$ and the corresponding $T_{c}^{*}$ (calc) were obtained by introducing the correction factor $\eta$ to the Roeser-Huber equation, which is listed as well. The parameter $\eta$ was obtained by adapting the calculation procedure manually to the experimentally obtained values of $T_{c}$. The result of this procedure is that we can now fully reproduce all the experimentally observed values for $T_{c}$. The slight deviations in $T_{\mathcal{C}}$ (calc) account for the difficulties when extracting the $T_{c}$-values. The data for the h-BN-WSe $e_{2}-\mathrm{tBLG}-\mathrm{h}-\mathrm{BN}$ stacks of Arora et al. [10] show that the WSe $\mathrm{W}_{2}$-layer stabilizes superconductivity at angles much smaller than the magic angle, and also smaller $\left(0.79^{\circ}\right)$ as the smallest angle reported for pure tBLG. We also see that such a monolayer of $\mathrm{WSe}_{2}$ is not superconducting on its own; Arora et al. describe the $\mathrm{WSe}_{2}$-layer as insulating. The trilayer graphene (TLG, Hao et al. [9]) would have a quite high $T_{c}$ of $8.5 \mathrm{~K}$ when calculating with $M_{L}=2$ due to the small value of $a_{M}$. Thus, the required $\eta$ is quite large and also off the fit in Fig. 3.

Figure 3 plots the resulting values for $\eta$ as function of temperature. The dashed green line indicates the bottom value of $\eta=2$, which corresponds to the case of HTSc materials. The lower the measured transition temperature, the larger the parameter $\eta$. Fitting the data with an exponential decay of the type

$$
y=2 A_{1} \cdot \exp \left(\left(x-x_{0}\right) / t_{1}\right),
$$




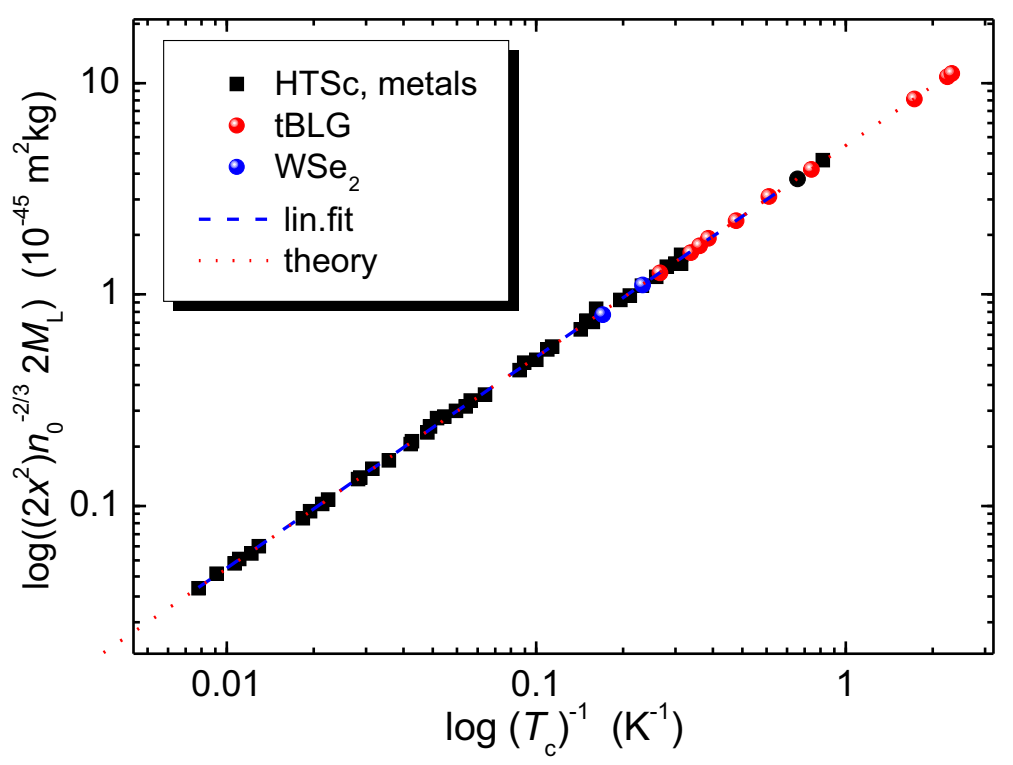

Figure 4. Roeser-Huber plot including the data of the various tBLG samples $(\bullet)$ and $\mathrm{WSe}_{2}(\bullet)$ and the previously calculated data for several HTSc and metals/alloys ( $\boldsymbol{\square})$. The straight red-dotted line follows the equation for a particle in a box [44] and the blue dashed line gives the linear fit to the data (see text).

we obtain a quite good correlation with the parameters $A_{1}=14.17, x_{0}=0.6$ and $t_{1}=$ 0.766 as shown in Fig. 3. The tBLG/WSe ${ }_{2}$-data fall below this fit line, and the TLG and $\mathrm{WSe}_{2}$ are located above it. Furthermore, the values for $\eta$ are only in a small range between 2 and 22, which is equal to the narrow window for the tBLG samples in the Uemura plot $\left(T_{c}\right.$ as a function of the Fermi temperature, $T_{F}=E_{F} / k_{B}$ with $E_{F}$ denoting the Fermi energy) in a line below the HTSc samples $[1,8]$. As $T_{F}$ is directly linked to the Fermi velocity, $v_{F}$, via

$$
T_{F}=\frac{m^{*} v_{F}^{2}}{2 k_{B}}
$$

and

$$
v_{F}=\frac{h}{2 \pi m_{e}}\left(2 \pi^{2} n\right)^{1 / 3},
$$

there is the effective mass, $m^{*}$, and the density of the charge carriers, $n$, directly involved. We also must note here that $\eta$ depends not only on the calculated value of $T_{c}$ at a given misorientation angle $\Theta$, but also on the charge carrier density. Thus, the parameter $\eta$ determined here should contain all this information, which will then also enable to judge via the value of $m^{*}\left(m^{*}<0.1 m_{e}\right)$ [8], if a material can be a superconductor or not. The newer experimental reports also present superconductivity measured at different superconducting domes in the phase diagram, which can be accessed at various positive and negative charge carrier densities. A dedicated analysis of all the data available (tBLG samples as well as the extreme elemental superconductors like Bi or Li) will allow to further clarify the properties of $\eta$.

The case of bi-layer $\mathrm{WSe}_{2}$ [11] is more complicated to be solved. The first problem in the case of $\mathrm{WSe}_{2}$ is the value for $n_{0}$ to be taken in the calculations. If a monolayer $\mathrm{WSe}_{2}$ is superconducting itself, $n_{0}$ must be taken as 2 . If only the product from two misaligned $\mathrm{WSe}_{2}$ layers is superconducting, we would have $n_{0}=1$ like for tBLG. A first glance on Table 1gives the idea that $n_{0}=2$ could be correct, but as seen from the combined WSe $e_{2}-\mathrm{tBLG}$-data from Arora et al. [10], we can consider $n_{0}=1$ to be the more realistic case. Thus, we have listed both cases in Table 1 to give some predictions of $T_{c}$ for the $\mathrm{WSe}_{2}$ system. As seen from Fig. 1c, the larger lattice parameter of $\mathrm{WSe}_{2}$ will lead to 
slightly larger $a_{M}$ for a given angle $\Theta$, and thus, the resulting values for $T_{\mathcal{C}}$ are higher as compared to tBLG, which is also observed experimentally [11]. The main problem is now that the experiments of Ref. [11] do not convincingly demonstrate superconductivity in this system as compared to the tBLG data, where much more detailed information is available. So it is difficult to extract properly defined values for $T_{C}$ from the data presented (WSe 2 bilayers with $1^{\circ}, 2^{\circ}$ and $4^{\circ}$ misalignment). For the $1^{\circ}$ sample (E7), $T_{\mathcal{C}}$ could be around 3.5-4 K, for the $2^{\circ}$ sample $\left(\mathrm{F}^{2}\right) \sim 4 \mathrm{~K}(-6.65 \mathrm{~V})$ or $\sim 6 \mathrm{~K}(-6.92 \mathrm{~V})$ and for the $4^{\circ}$ sample (D11, marked by a star in Table 1$)$, one may get $T_{\mathcal{C}}$ somewhere between 4 $\mathrm{K}$ and $12 \mathrm{~K}$, if at all. The calculation of the Moiré pattern parameter for the $4^{\circ}$ sample gives $a_{M}=4.72 \mathrm{~nm}$, which would yield a $T_{\mathcal{c}}$ of $49.9 \mathrm{~K}$ (with $n_{0}=2$ ) or $31.13 \mathrm{~K}$ with $n_{0}=$ 1 . These values for $\Delta_{(0)}$ and $T_{\mathcal{C}}$ are considerably too high and unrealistic. As the authors show in their paper higher order Laue reflexes from electron diffraction patterns for the $1^{\circ}$ sample, which would indicate a lattice constant of the order of 20-25 nm (instead of the calculated $18.9^{c}$ irc using eq. (5)), we have used $20 \mathrm{~nm}$ for $x$ in Table 1 for the $1^{\circ}$ sample and left the $4^{\circ}$ sample out of further consideration. If we calculate $T_{c}$ using $n_{0}=$ 2 , the calculated values come quite close to the experimental data assuming $T_{\mathcal{C}} \sim 3 \mathrm{~K}$. In all cases, the superconductivity is best documented for sample $\mathrm{F}^{2}$ (their Figs. 5a and S11), yielding a $T_{c}$ of $4.53 \mathrm{~K}(-6.65 \mathrm{~V})$ and $6.1(-6.92 \mathrm{~V})$ at two different gate voltages. These $T_{c}$-values are clearly higher than those of tBLG, but also smaller than the calculated value of $7.78 \mathrm{~K}\left(n_{0}=1\right)$. Determining the correction factors $\eta$ for this sample yields $\eta=$ 3.44 and 2.55 at the two gate voltages, which are only small corrections. To summarize this part, the published data of $\mathrm{WSe}_{2}$ are not suitable for a good comparison, but when extracting $T_{C}$ via the first derivative from the published data (best for sample $\mathrm{F}^{2}$ ), we only require small correction factors to reproduce the experimental $T_{c}$. This would indicate that the $\mathrm{WSe}_{2}$ bilayers have properties being more similar to that of HTSc compounds.

Finally, Fig. 4 presents the Roeser-Huber plot, now extended towards lower temperatures to include the various tBLG samples reported in the literature. The black

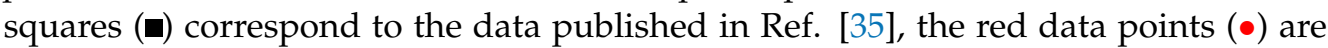
for the various $\mathrm{tBLG}$ samples investigated here, and the blue bullet $(\bullet)$ shows the $2^{\circ}$ $\mathrm{WSe}_{2}$ data. The straight red-dotted line follows the equation for a particle in a box [44] with the slope $h^{2} /\left(2 \pi k_{B}\right)=5.061 \times 10^{-45} \mathrm{~m}^{2} \mathrm{~kg} \mathrm{~K}$. The linear fit (dashed-blue line) is almost perfect with only a small error margin, which manifests the basic idea of the Roeser-Huber formalism.

Harshman and Fiory [45] presented another way of calculating the transition temperature of $\mathrm{tBLG}$ from experimental data. Also this approach was originally developed for HTSc samples, and the parameters involved are quite similar to those of the RoeserHuber approach. However, there is no relation between the $T_{C}$ and the crystal lattice parameters, except a distance between the superconducting layers, which in turn is not contained in the Roeser-Huber formalism. In all cases, it will be interesting to compare the various parameters of the models with each other.

So, we can say here that an extension of the Roeser-Huber formula is required to account for the low charge carrier densities and the resulting low charge carrier mass using the new parameter $\eta$. When doing so, we can directly reproduce the experimental data of the various $\mathrm{tBLG}$ measurements published in the literature. We also note that the calculation using $\eta=2$, that is, a charge carrier mass of $2 m_{e}$, gives an upper limit for $T_{\mathcal{C}}$, to which the experiments come now close by applying pressure or using thicker h-BN layers. This observation is a very positive output for use of the Roeser-Huber equation to predict the transition temperatures of still unknown materials.

\section{Conclusion}

To conclude, we have shown the calculation of $T_{c}$ of Moiré superconductors based on the Moiré lattice parameter. The Roeser-Huber formula in the standard form with $M_{L}=2 m_{e}$ gives an upper limit of $T_{\mathcal{C}}$ for tBLG, which is close to the experimental 
observations. Introducing a correction factor $\eta$ to the Roeser-Huber formalism enables to account for the small charge carrier density and charge carrier mass, so that the experimentally obtained data can successfully be reproduced. Further work is required to find a theoretical foundation for $\eta$. For WSe $e_{2}$, the currently available experimental data are not sufficient to extract proper values for $T_{\mathcal{C}}$ to enable a proper comparison with calculated data.

Author Contributions: Conceptualization, M.R.K.; Formal Analysis, A.K.-V. and M.R.K.; Investigation, A.K.-V. and M.R.K.; WritingOriginal Draft Preparation, M.R.K.; Writing-Review and Editing, A.K.-V. and M.R.K.

Funding: This work is part of the SUPERFOAM international project funded by ANR and DFG under the references ANR-17-CE05-0030 and DFG-ANR Ko2323-10, respectively.

Conflicts of Interest: The authors declare no conflict of interest.

\section{References}

1. Cao, Y.; Fatemi, V.; Fang, S.; Watanabe, K.; Taniguchi, T.; Kaxiras, E.; Jarillo-Herrero, P. Unconventional superconductivity in magic-angle graphene superlattices. Nature 2018 556, 43-50; doi:10.1038/nature26160.

2. Yankowitz, M.; Chen, S.; Polshyn, H.; Zhang, Y.; Watanabe, K.; Taniguchi, T.; Graf, D.; Young, A. F.; Dean, C. R. Tuning superconductivity in twisted bilayer graphene. Science 2019 363, 1059-1064; doi: 10.1126/science.aav1910.

3. Lu, X.; Stepanov, P.; Yang, W.; Xie, M.; Ali Aamir, M.; Das, I.; Urgell, C.; Watanabe, K.; Taniguchi, T.; Zhang, G.; Bachtold, A.; MacDonald, A. H.; Efetov, D. K. Superconductors, orbital magnets and correlated states in magic-angle bilayer graphene. Nature 2019 574, 653-657; doi: 10.1038/s41586-019-1695-0.

4. Codecido, E.; Wang, Q.; Koester, R.; Che, S.; Tian, H.; Lv, R.; Tran, S.; Watanabe, K.; Taniguchi, T.; Zhang, F.; Bockrath, M.; Lau, C. N. Correlated insulating and superconducting states in twisted bilayer graphene below the magic angle. Sci. Adv. 2019 5, eaaw9770; doi: 10.1126/sciadv.aaw9770.

5. Liu, X.; Hao, Z.; Khalaf, E.; Lee, J.-Y.; Watanabe, K.; Taniguchi, T.; Vishwanath, A.; Kim, P. Tunable spin-polarized correlated states in twisted double bilayer graphene. Nature 2020 583, 221-225; doi:10.1038/s41586-020-2458-7.

6. Stepanov, P.; Das, I.; Lu, X.; Fahimniya, A.; Watanabe, K.; Taniguchi, T.; Koppens, F. H. L.; Lischner, J.; Levitov, L.; Efetov, F. K. Untying the insulating and superconducting orders in magic-angle graphene. Nature 2020 583, 375-378; doi: 10.1038/s41586-0202459-6.

7. Saito, Y.; Ge, J.; Watanabe, K.; Taniguchi, T.; Young, A. F. Independent superconductors and correlated insulators in twisted bilayer graphene Nature Phys. 2020 16, 926-930; doi: 10.1038/s41567-020-0928-3.

8. E. F. Talantsev, E.. F.; Mataira, R. C.; Crump, W. P. Classifying superconductivity in Moiré graphene superlattices. Sci. Rep. 2020 10, 212; doi: 10.1038/s41598-019-57055-w.

9. Hao, Z.; Zimmerman, A. M.; Ledwith, P.; Khalaf, E.; Najafabadi, D. H.; Watanabe, K.; Taniguchi, T.; Vishwanath, A.; Kim, P. Electric field-tunable superconductivity in alternating-twist magic-angle trilayer graphene. Science 2021 371, 1133-1138; doi: 10.1126/science.abg0399.

10. Arora, H. S.; Polski, R.; Zhang, Y.; Thomson, A.; Choi, Y.; Kim, H.; Lin, Z.; Wilson, I. Z.; Xu, X.; Chu, J.-H.; Watanabe, K.; Taniguchi, T.; Alicea, J.; Nadj-Perge, S. Superconductivity in metallic twisted bilayer graphene stabilized by WSe2. Nature 2020 583, 379-384; doi: 10.1038/s41586-020-2473-8.

11. An, L.; Cai, X.; Pei, D.; Huang, M.; Wu, Z.; Zhou, Z.; Lin, J.; Ying, Z.; Ye, Z.; Feng, X.; Gao, R.; Cacho, C.; Watson, M.; Chen, Y.; and Wang, N. Interaction effects and superconductivity signatures in twisted double-bilayer WSe 2 . Nanoscale Horiz. 2020 5, 1309 ; doi: 10.1039/d0nh00248h.

12. Balents, L.; Dean, C. R.; Efetov, D. K.; Young, A. F. Superconductivity and strong correlations in moiré flat bands. Nature Phys. 2020 16, 725-733; doi: 10.1038/s41567-020-0906-9.

13. Bistritzer, R.; MacDonald, A. H. Moiré bands in twisted double-layer graphene. Proc. Natl. Acad. Sci. 2011 108, 12233-12237; doi: 10.1073/pnas.1108174108.

14. Wu, F.; Hwang, E.; Das Sarma, S. Phonon-induced giant linear-in-T resistivity in magic angle twisted bilayer graphene: Ordinary strangeness and exotic superconductivity. Phys. Rev. B 2019 99, 165112; doi: 10.1103/PhysRevB.99.165112.

15. Schrade, C.; Fu, L. Spin-valley density wave in moiré materials. Phys. Rev. B 2019 100, 035413; doi: 10.1103/PhysRevB.100.035413.

16. Shi, L.; Ma, J., Song, J. C. W. Gate-tunable flat bands in van der Waals patterned dielectric superlattices. 2D Mater. 2020 7, 015208 ; doi: $10.10 \ldots . .$.

17. Decker, R.; Wang, Y.; Brar, V.W.; Regan, W.; Tsai, H.-Z.; Wu, Q.; Gannett, W.; Zettl, A.; Crommie, M. F. Local Electronic Properties of Graphene on a BN Substrate via Scanning Tunneling Microscopy. Nano Lett. 2011 11, 2291-2295; doi: 10.1021/nl2005115.

18. Wang, L.; Zihlmann, S.; Liu, M.-H.; Makk, P.; Watanabe, K.; Taniguchi, T.; Baumgartner, A.; Schönenberger, Chr. New Generation of Moiré Superlattices in Doubly Aligned hBN/Graphene/hBN Heterostructures. Nano Lett. 2019 19, 2371-2376; doi: 10.1021/acs.nanolett.8b05061.

19. Hermann, K. Periodic overlayers and moiré patterns: theoretical studies of geometric properties. J. Phys.: Condens. Matter 2012 24, 314210; doi: 10.1088/0953-8984/24/31/314210. 
20. Moon, P.; Koshino, M. Electronic properties of graphene/hexagonal-boron-nitride moiré superlattice. Phys. Rev. B 2014 90, 155406; doi: 10.1103/PhysRevB.90.155406.

21. Anđelković, M.; Milovanovic, S. P.; Covaci, L.; Peeters, F. M. Double moiré with a twist: super-moiré in encapsulated graphene. Nano Lett. 2020 (), 9b04058; doi:10.1021/acs.nanolett.9b04058.

22. Tang, K.; Qi, W. Moiré-Pattern-Tuned Electronic Structures of van der Waals Heterostructures. Adv. Funct. Mater. 2020 2020, 2002672; doi: 10.1002/adfm.202002672.

23. Martin, I. Moiré superconductivity. Annals of Physics 2020 417, 116118; doi: 10.1016/j.aop.2020.168118.

24. Stanev, V., Oses, C., Kusne, A. G., Rodriguez, E., Paglione, J., Curtarolo, S., Takeuchi, I. Machine learning modeling of superconducting critical temperature. npj Computational Materials 2018 4, 29, doi:10.1038/s41524-018-0085-8.

25. Matsumoto, K., Horide, T. An acceleration search method of higher $T_{\mathcal{c}}$ superconductors by a machine learning algorithm. Appl. Phys. Express 2019 12, 073003, doi: 10.7567/1882-9786/ab2822.

26. Hutcheon, M. J.; Alice M. Shipley, A. M.; Richard J. Needs, R. J. Predicting novel superconducting hydrides using machine learning approaches. Phys. Rev. B 2020 101, 144505; doi: 10.1103/PhysRevB.101.144505.

27. Lee, D.; You, D.; Lee, D.; Li, X.; Kim, S. Machine-Learning-Guided Prediction Models of Critical Temperature of Cuprates. J. Phys. Chem. Lett. 2021 12, 6211-6217; doi: 10.1021/acs.jpclett.1c01442.

28. Zeng, S.; Zhao, Y.; Li,G.; Wang, R.; Wang, X.; Ni, J. Atom table convolutional neural networks for an accurate prediction of compounds properties. npj Computational Materials 2019 5, 84; https:/ / doi.org/10.1038/s41524-019-0223-y.

29. Roeser, H. P., Hetfleisch, Huber, F. M., Stepper, M., von Schoenermark, M. F., Moritz, A., Nikoghosyan, A. S. A link between critical transition temperature and the structure of superconducting $\mathrm{YBa}_{2} \mathrm{Cu}_{3} \mathrm{O}_{7-\delta}$. Acta Astronautica 2008 62, 733-736; doi: 10.1016/j.actaastro.2008.04.004.

30. Roeser, H. P., Hetfleisch, Huber, F. M., von Schoenermark, M. F., Stepper, M.; Moritz, A., Nikoghosyan, A. S. Correlation between oxygen excess density and critical temperature in superconducting Bi-2201, Bi-2212 and Bi-2223. Acta Astronautica 2008 63, 1372-1375; doi:10.1016/j.actaastro.2008.06.001.

31. Roeser, H. P., Huber, F. M., von Schoenermark, M. F., Nikoghosyan, A. S. High temperature superconducting with two doping atoms in La-doped Bi-2201 and Y-doped Bi-2212. Acta Astronautica 2009 65, 489-494, doi:10.1016/j.actaastro.2009.02.004.

32. Roeser, H. P., Haslam, D. T., Hetfleisch, F., Lopez , J. S., von Schoenermark, M. F., Stepper, M., Huber, F. M., Nikoghosyan, A. S. Electron transport in nanostructures: A key to high temperature superconductivity? Acta Astronautica 2010 67, 546-552, doi:10.1016/j.actaastro.2010.04.014.

33. Roeser, H.P.; Bohr, A.; Haslam, D.T.; López, J.S.; Stepper, M.; Nikoghosyan, A.S. Size quantization in high-temperature superconducting cuprates and a link to Einstein's diffusion law. Acta Astronaut. 2012, 76, 37-41, doi:10.1016/j.actaastro.2012.04.014.

34. Huber, F.; Roeser, H. P., von Schoenermark, M. A correlation between $T_{c}$ of Fe-based HT Superconductors and the crystal super lattice constants of the doping element positions. J. Phys. Soc. Jpn. 200877 Suppl. C, 142-144; doi: 10.1143/JPSJS.77SC.142.

35. Koblischka, M. R., Roth, S., Koblischka-Veneva, A., Karwoth, T., Wiederhold, A., Zeng, X. L., Fasoulas, S., and Murakami, M. Relation between Crystal Structure and Transition Temperature of Superconducting Metals and Alloys. Metals 2020 10, 158; doi: $10.3390 /$ met10020158.

36. Koblischka-Veneva, A., Koblischka, M. R. (RE)BCO and the Roeser-Huber formula. Materials 2021 14, 6068; doi: 10.3390/ma14206068

37. Ghosh, K. J. B.; Kais, S.; Herschbach, D. R. Dimensional interpolation for metallic hydrogen. Phys. Chem. Chem. Phys. 2021 23, 7841-7848; doi: 10.1039/d0cp05301e.

38. Mori, N.; Wilson, J. A.; Ozaki, H. Fluctuation conductivity in the 110-K phase of Ni-doped (Bi,Pb)-Sr-Ca-Cu-O superconductors. Phys. Rev. B 1992 45, 10633-10638; doi: 10.1103/PhysRevB.45.10633.

39. Larkin, A.; Varlamov, A. Fluctuation Phenomena in Superconductors. Oxford University Press, Oxford, U.K., 2005.

40. Koblischka, M. R.; Koblischka-Veneva, A.; Zeng, X. L.; Hannachi, E.; Slimani, Y. Microstructure and Fluctuation-Induced Conductivity Analysis of $\mathrm{Bi}_{2} \mathrm{Sr}_{2} \mathrm{CaCu}_{2} \mathrm{O}_{8+\delta}$ (Bi-2212) Nanowire Fabrics. Crystals 2020 10, 986; doi:10.3390/cryst10110986.

41. Uemura, Y. J.; Le, L. P.; Luke, G. M.; Sternlieb, B. J.; Wu, W. D.; Brewer, J. H.; Riseman, T. M.; Seaman, C. L.; Maple, M. B.; Ishikawa, M.; Hinks, D. G.; Jorgensen, J. D.; Saito, G.; Yamochi, H. Basic Similarities among Cuprate, Bismuthate, Organic, Chevrel-Phase, and Heavy-Fermion Superconductors Shown by Penetration Depth Measurements. Phys. Rev. Lett. 1991 66, 2665-2668; doi: 10.1103/PhysRevLett.66.2665.

42. Uemura, Y. J. Condensation, excitation, pairing, and superfluid density in high- $T_{\mathcal{C}}$ superconductors: the magnetic resonance mode as a roton analogue and a possible spin-mediated pairing. J. Phys.: Condens. Matter 16 (2004) S4515-S4540; doi: 10.1088/0953-8984/16/40/007.

43. Mathew, S.; Aben Regi Abraham; Chintalapati, S.; Sarkar, S.; Joseph, B.; Venkatesan, T. Temperature Dependent Structural Evolution of $\mathrm{WSe}_{2}$ : A Synchrotron X-ray Diffraction Study. Condens. Matter 2020 5, 76; doi:10.3390/condmat5040076.

44. Rohlf, J. W.: Modern Physics from $\alpha$ to $Z^{0}$. Wiley, New York (1994).

45. Harshman, D. R.; Fiory, A. T. High- $T_{c}$ Superconductivity Originating from Interlayer Coulomb Coupling in Gate-Charged Twisted Bilayer Graphene Moiré Superlattices. J. Supercond. 2020 33, 367-378; doi: 10.1007/s10948-019-05183-9. 